\title{
How robust is a neural circuit?
}

\author{
PETER STERLING AND MICHAEL FREED \\ Department of Neuroscience, University of Pennsylvania, Philadelphia, Pennsylvania
}

(Received March 12, 2007; AcCePTEd May 23, 2007)

\begin{abstract}
Design in engineering begins with the problem of robustness - by what factor should intrinsic capacity exceed normal demand? Here we consider robustness for a neural circuit that crosses the retina from cones to ganglion cells. The circuit's task is to represent the visual scene at many successive stages, each time by modulating a stream of stochastic events: photoisomerizations, then transmitter quanta, then spikes. At early stages, the event rates are high to achieve some critical signal-to-noise ratio and temporal bandwidth, which together set the information rate. Then neural circuits concentrate the information and repackage it, so that nearly the same total information can be represented by modulating far lower event rates. This is important for spiking because of its high metabolic cost. Considering various measurements at the outer and inner retina, we conclude that the "safety factors" are about $2-10$, similar to other tissues.
\end{abstract}

Keywords: Retina, Circuitry, Information, Synapse, Spikes

To design a bridge an engineer must first know its intended use-for that determines the necessary level of performance. One design would serve civilian commuter traffic, the 2-ton family SUV, but quite a different design would be needed for military traffic, the 30 ton Bradley armored vehicle. After considering the most probable loads and the physical limits of the construction materials, the engineer must decide how robustly to build and choose a margin of safety. If the bridge can bear a 100-fold greater load than the one it will normally encounter, it will rarely collapse. But robustness has a cost, and the Econo Construction Corp, competing with Cutthroat Builders, would order the engineer to thin down the cables and lengthen the spans, reducing the safety margin to the minimum acceptable to the Grim Casualty Insurance Corp. Thus performance and robustness are set by a corporation to maximize its competitive position. Natural selection, in designing organs and tissues, is equally unsentimental: it too constrains these factors by cost to maximize fitness (Diamond, 1993).

Safety factors are known for various biological tissues, such as muscle, bone, small intestine, and lung. They are generally on the order of 2 to 10-fold (Diamond, 1993). Furthermore, efficient biological design matches the capacity of each tissue to the others to which it is functionally coupled (Weibel, 2000). Biological design has the further advantage that, as demand shifts on some appropriate time scale, functional capacities adjust to match the new level; i.e., they adapt (Fig. 1). For example, as muscle strengthens with exercise, bone thickens correspondingly (Alex-

Address correspondence and reprint requests to: Peter Sterling, Department of Neuroscience, University of Pennsylvania, 123 Anatomy/Chemistry Building, 37th \& Service Drive, Philadelphia, PA 19104-6058. E-mail: peter@retina.anatomy.upenn.edu ander, 1996). Thus adaptation holds robustness constant across a range of demand so that the organism is never overbuilt and pays only for what it uses. A bridge designer should live so long!

Here we treat a neural bridge across the retina-whereby cones connect via bipolar neurons to ganglion cells. This bridge conducts information, at the entrance by modulating transmitter quanta from cone synapses, and at the exit by modulating spikes from ganglion cells. As we shall explain, there a striking transformation: whereas information approaching the bridge requires a continuous stream of quanta at high mean rates, the same information leaving the bridge is represented by transient bursts of quanta at astonishingly low mean rates, and finally by spikes at still lower mean rates. Since total information is strongly conserved (Savage \& Banks, 1992; Borghuis, Smith \& Sterling, unpublished), one expects robustness at all stages. By exploring the question of robustness, we also learned something about why the bridge is needed.

\section{Robustness at retinal synapses}

Issues of cost are manifest in the design of neural circuits, which are limited by energy and space. Consider space: the human skull allots the brain only about $1500 \mathrm{~cm}^{3}$, and a presynaptic terminal occupies a certain, irreducible volume $\left(\sim 1 \mu \mathrm{m}^{3}\right)$, which must accommodate sufficient synaptic vesicles to meet its primary function, i.e., release transmitter quanta on demand. To increase capacity for release, terminals might store more vesicles, but this would increase their volumes, and necessarily decrease their numbers because theoretical studies show that the ratio of terminal volume to "wire" volume is fixed (Chklovskii et al., 2002). Thus there must be selective pressure to match presynaptic volume to release rate, leading one to wonder: just how robust is a single 


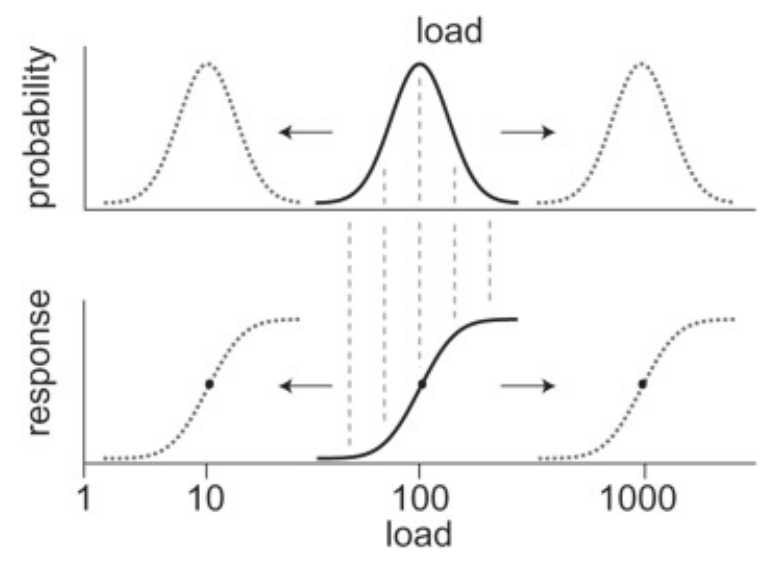

Fig. 1. Adaptive mechanisms shift the input/output curve to hold it centered on the most probable load (after Laughlin, 1981). This allows the "safety factor" to remain constant across sustained changes in load.

presynaptic terminal? That is, how long could the terminal release without exhausting its supply of vesicles?

There must also be selective pressure to match the machinery for vesicular release to the required information rate. Presumably the machinery for release-ribbons, molecular motors, tethering proteins, etc-is energetically expensive to maintain and bulky, and so an overcapacity for release would be wasteful. The question arises: how does the capacity for release compare to the actual rate of release under natural conditions? That is, what is the ratio of capacity to normal load?

Capacity for continuous release over minutes has been evaluated for cones by bathing the retina in a fluorescent dye that stains vesicles, then stimulating release and observing the course of destaining (Choi et al., 2005; Rea et al., 2004). Capacity for transient release over 100s of milliseconds was accomplished initially for the "giant" terminal of the goldfish ON bipolar cell by intensely depolarizing an isolated neuron, and then detecting vesicle fusion as a jump in membrane capacitance (Neves \& Lagnado, 1999; von Gersdorff \& Matthews, 1994). Fusion has also been detected optically as an expansion of the terminal's "footprint" (Llobet et al., 2003) and further confirmed by detecting release using a "sniffer" neuron (von Gersdorff et al., 1998). Now there are similar measurements from rodent bipolar terminals (Singer \& Diamond, 2006; Zhou et al., 2006). These experiments are technical tours-de-force, but conceptually simple: isolate a single cell, stimulate it maximally, and measure the rate of vesicle release.

Measuring release rate under natural conditions is a messier affair: it requires that the retina be intact and responsive to natural stimuli. Furthermore, quantal release cannot be measured directly from a single terminal in the intact retina. So one must record postsynaptic responses from a bipolar or ganglion cell, and because quanta arrive synchronously in clumps, tease out the number of quanta in a clump using "noise analysis." Finally, to learn the rate at a single active zone, one needs to determine the number of presynaptic contacts. Sufficient data on these points has now accumulated to reasonably evaluate robustness.

It turns out that robustness in the circuit between photoreceptors and ganglion cells is similar to what has been found for other tissues and systems. A cone terminal can release for 3-10 min without resupply of vesicles. Furthermore, a cone's capacity for continuous release over minutes is about twice its capacity, a safety factor fairly common in biology. But astonishingly, a fully depolarized bipolar ribbon synapse can release 2500-fold more vesicles in a $100 \mathrm{~ms}$ burst than can naturalistic stimulation. Such apparently immense robustness begs for explanation-requiring a broader view that asks why the representation of information should be transformed on its passage from cone to ganglion cell, i.e., what is the actual purpose of the neural bridge?

\section{Information packets at the cone}

The cone outer segment integrates photoisomerizations $\left(\mathrm{R}^{*}\right)$ across a narrow spatial aperture (a few microns in diameter) and a time window of about $100 \mathrm{~ms}$. Reasonably strong daylight (say, $10^{4}$ $\mathrm{R}^{*} / \mu \mathrm{m} 2 / \mathrm{s}$ ) provides $10^{3} \mathrm{R}^{*}$ in this time window, but daytime $\mathrm{R}^{*}$ rates vary tremendously-over six log units. On the other hand, the cone photovoltage can be modulated only over a few tens of millivolts; so to use the linear region of a steep intensity/voltage curve, the cone must adapt (as cartooned in Fig. 1). This "intensity" adaptation apparently occurs in the outer segment and is completed within the cone integration time. Adaptation is evident at the first postsynaptic cells (Burkhardt, 2001). Fly photoreceptors face the same problem, matching a limited dynamic range to a large range of intensities, and also solve it by adaptationalthough by different subcellular mechanisms (Laughlin \& Hardie, 1978; Laughlin, 1994).

The finely modulated photovoltage arriving at the cone synaptic terminal is next represented by modulating a stochastic stream of transmitter quanta. Without some type of image compression representation would require at least as many transmitter quanta as the particles $\left(\mathrm{R}^{*}\right)$ that originally produced it, on the order of $10^{3}$ vesicles over $100 \mathrm{~ms}$. So, before transmission, the image is compressed by removing correlations - on principles familiar from digital photography. Photovoltages in adjacent cones (pixels) are correlated because of local correlations within a scene and by optical blur. To remove correlations, the circuitry implements a "background subtraction" algorithm (high-pass filter). Horizontal cells take a broad spatio-temporal average and subtract it from each cone terminal via negative feedback. Stripping away the original information about mean light intensity leaves information primarily representing contrast. The signal to be quantized is thereby compressed from a mean of $10^{4} \mathrm{R}^{*} / 100 \mathrm{~ms}$ and a range of $6 \log$ units to a mean of 0 with a range of about $2 \log$ units (Laughlin, 1981; Ratliff, Kao, Sterling \& Balasubramanian, unpublished; Richards, 1981; Srinivasan et al., 1982). Thus, the same total information can now be represented by modulating a much smaller stream of stochastic events.

Before transmission, the cone terminal also removes noise due to photon and channel fluctuations that would otherwise appear in the cone photovoltage. Gap junctions between the cone synaptic terminals pool these voltages and thereby improve signal-to-noise, thus implementing a low-pass filter. This extends the cone receptive field considerably beyond its optical aperture-by $50 \%$ for a foveal cone (DeVries et al., 2002) and by about 10-fold (to $\sim 50 \mu \mathrm{m}$ ) for nonfoveal cones (Nelson, 1977; Smith \& Sterling, 1990). Here there is resemblance to the fly, whose six photoreceptors contacting a bipolar-like neuron register identical optical images (Laughlin, 1994).

\section{Quantizing the contrast signal: Robustness of tonic release from the cone terminal}

The continuous release rate from a lizard cone in the intact retina has been measured over minutes by measuring exocytotic release 
of FM1-43 dye. When the cone is maximally depolarized by placing the retina in high $\mathrm{K}^{+}$, it releases 24000 vesicles/min, but when the retina is placed in darkness, the cone releases only about 15000 vesicles/min (Fig. 2). Thus, taking the $\mathrm{K}^{+}$-stimulated rate as capacity and the dark rate as the maximal load, the safety factor over minutes is about 2. As light intensity increases, release declines below the dark rate and thus always stays well within this safety factor. Under photopic illumination $\left(10^{4} \mathrm{R} * / \mu \mathrm{m}^{2} / \mathrm{s}\right)$, the mean release rate declines to 9600 vesicles per minute. Although brief reductions in contrast would cause transient increases in release that might exceed the continuous dark rate, a photoreceptor's capacity can momentarily surge to meet this demand. For example, an amphibian rod can at release 17500 vesicles/s, but this extreme capacity can only be sustained for $200 \mathrm{~ms}$ (Thoreson et al., 2004).

To support its continuous dark rate, the lizard cone terminal maintains a population of about 170000 vesicles (Choi et al., 2005). Of these about 145000 diffuse freely within the terminal and participate in the cycle of exo- and endocytosis (Rea et al., 2004). If this cycle had no lag, such a large inventory of vesicles would be unnecessary. By analogy, Toyota maintains a "just-intime" parts supply for its assembly lines and thus avoids large warehouses required by other manufacturers. Thus the safety margin for release is the size of the vesicle inventory divided by the number of vesicles released during the lag, i.e., the interval between release and restocking.

The lag time is the time needed for a fused vesicle to be retrieved, refilled, and reprimed for release. Measurements in goldfish bipolar cell (Lagnado et al., 1996) and calculations from
FM1-43 uptake in lizard cone (Rea et al., 2004), suggest that a vesicle requires about a minute to become re-releasable. During this lag, the dark rate would release about 15000 vesicles. Thus the store of 145000 releasable vesicles provides a safety margin of about $10 \mathrm{~min}$.

The maximum rate for mammalian cones has not been measured directly but has been estimated from recordings from the postsynaptic bipolar cell. When a cone in ground squirrel is depolarized sufficiently to release at its peak rate, a postsynaptic cone bipolar cell receives about 700 quanta/s from about seven invaginations (DeVries et al., 2006). Since each cone contains about 20 invaginations (Li \& DeVries, 2006), this suggests that a mammalian cone releases maximally about 2000 vesicles/s, considerably more than the lizard cone. In the dark, the mammalian cone probably releases about two-thirds of this maximal rate or about 1300 vesicles/s, and during photopic illumination, very approximately one-third, or 700 vesicles/s (Fig. 2; Choi et al., 2005). Thus, compared to the rate of photon capture, the rate of quanta leaving the cone is on the order of 10 -fold lower. At higher light intensities this ratio becomes still larger.

Consistent with its higher release rate, the mammalian cone terminal contains more vesicles; e.g., a primate foveal cone contains about 234000 vesicles (Bronk \& Sterling, unpublished). If the same fraction were releasable as in the lizard, 200000 vesicles would be cycling. Thus during a minute lag at the dark rate $(1300 / \mathrm{s})$, about 80000 vesicles would be released. Thus the store of vesicles provides a safety margin of nearly $3 \mathrm{~min}$.

Another measure of robustness relates to the number of vesicles docked along the base of the ribbon and tethered to the face. These

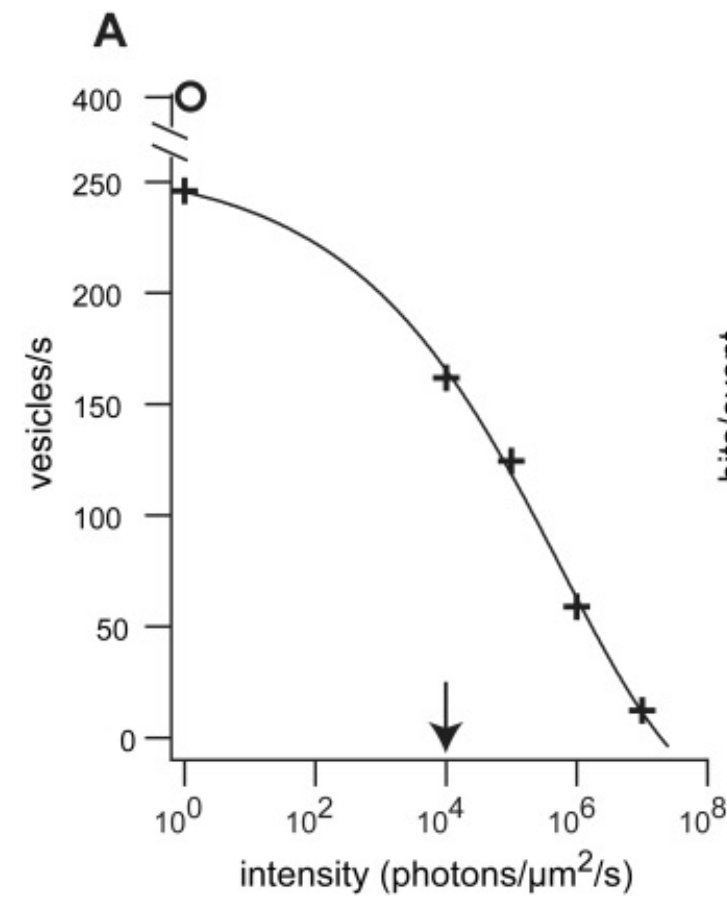

B

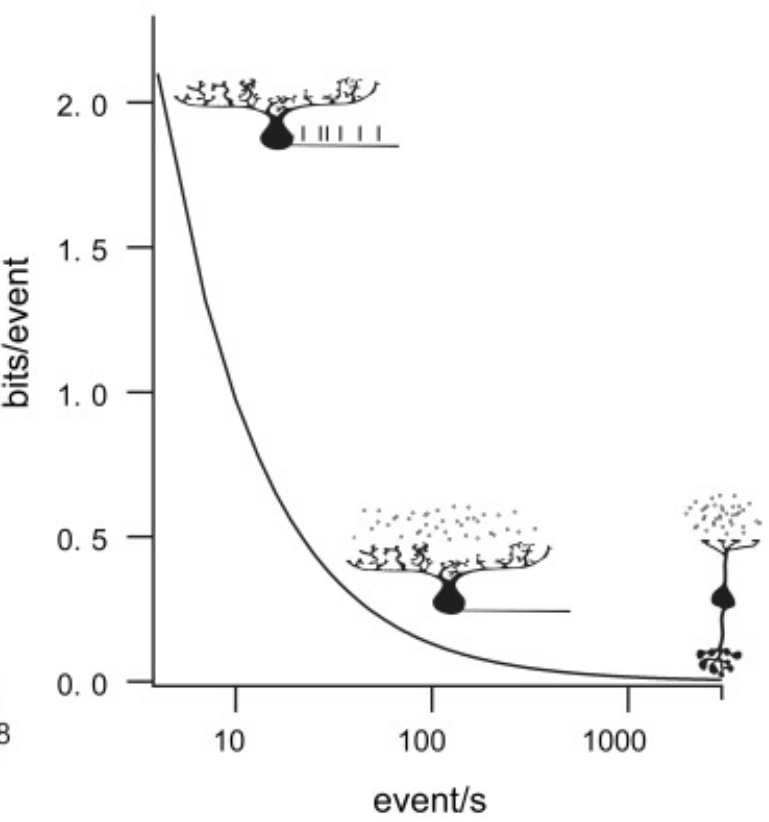

Fig. 2. (A) Vesicle release rates from cone decrease with light intensity. Release was estimated in lizard cones as loss of fluorescence when vesicles labeled by FM1-43 dye fuse to the plasma membrane during exocytosis (after Choi et al., 2005). The solid curve is a sigmoidal fit to the estimated release rates $(+)$. The maximum release rate $(\mathrm{O})$ was evoked by high potassium; arrow marks mean photopic intensity used in all calculations, both in part B and in text. (B) In a Poisson stream of events, information content of each event declines logarithmically with event rate. Events are (from left to right): 4 spikes/s fired by ganglion cell (Koch et al., 2006); 100 quanta/s received by ganglion cell (Freed, 2005); 7000 quanta/s received by bipolar cell from 10 cones (estimated from DeVries et al., 2006). Solid curve is from equation 3, assuming an initial information rate for the ganglion cell of 8 bits/s (Koch et al., 2004). 
numbers indicate how long a ribbon could release vesicles at a given rate without being resupplied. Terminals in the central retina of cat and monkey contain 12-20 ribbons whose total length is about $10 \mu \mathrm{m}$ (Esfahani et al., 1993; Haverkamp et al., 2000; Sterling, 2004). With vesicles spaced at $70 \mathrm{~nm}$ between centers (Raviola, 1976), about 286 vesicles would be docked. Ribbons in monkey cone are $0.3 \mu \mathrm{m}$ high (Esfahani et al., 1993), so in that dimension a ribbon accommodates about 4.3 vesicles and the two faces of all the ribbons thus tether about 2560 vesicles. Total vesicles docked and tethered for a central cone would be about 2750. Assuming a mammalian dark rate (1300 vesicles/s), the cone ribbons can release without resupply for about $2 \mathrm{~s}$.

\section{Why are bipolar cells needed?}

Bipolar cells form the bridge connecting cones to ganglion cells_-of course. But what is the essential nature of the chasm that is thereby spanned? Why not simply deliver the visual image-which is fully represented in the stream of quanta from a mammalian cone (estimated at 700/s under photopic illumination) - directly to ganglion cells? Consider that the ganglion cell represents the same information in yet another stream, a train of action potentials. But action potentials are metabolically expensive (Attwell \& Laughlin, 2001), so costly in fact that the human cerebral cortex can afford less than one spike/cell/s (Lennie, 2003).

Thus, we propose that a key task of retinal circuitry is to repackage information represented at high rates in the cone's quantal stream so that ganglion cells can send it at much lower rates (Fig. 2). For example, in photopic conditions $3 \times 10^{6}$ cones in guinea pig retina would release about $2 \times 10^{9}$ transmitter quanta/s. The guinea pig's 100000 ganglion cells fire overall about $5 \times 10^{5}$ spikes/s (calculated from Tables 1 and S2 in Koch et al., 2006). Therefore, the bipolar arrays must rigorously condense and rearrange the information, so that the mean event rate can be reduced between input and output streams by about 4000 -fold.

When information is represented by modulating a Poisson stream, information conveyed by each event declines at higher rates because of redundancy between events (de Ruyter van Steveninck \& Laughlin, 1996). How greatly information declines can be roughly estimated by assuming that all quanta carry the same signal plus some noise. As the quantal rate increases by a factor of $n$, the signal-to-noise ratio (SNR) improves less than proportionately:

$$
S N R \propto \sqrt{n}
$$

Thus according to Shannon's equation for a Gaussian channel, assuming a fixed bandwidth and SNR $>1$, information will improve only incrementally by

$$
\Delta I=\log _{2}(n) \text { bits } / \mathrm{s} .
$$

Thus, if an event's initial information content is $I_{1}$ and a rate of $n_{1}$, after the rate increases to $n_{2}$, its final information content is:

$$
I_{2}=\frac{I_{1} n_{1}+\log _{2}\left(\frac{n_{2}}{n_{1}}\right)}{n_{2}} \text { bits. }
$$

To see how this equation plays out, it is convenient to start at the bridge exit and work toward the entrance. Under photopic condi- tions, the ganglion cell fires about 4 spikes/s $\left(=n_{1}\right)$, each spike carrying about 2 bits $\left(=I_{1}\right.$ ) (Koch et al., 2004, 2006). At the middle of the bridge, cone bipolar cells provide a brisk-sustained ganglion cell with about 100 quanta/s; the equation indicates that each quantum should represent about 0.13 bits, roughly what has been estimated experimentally (0.1-0.4 bits; Freed, 2005). At the bridge entrance, we estimated above that a cone releases about 700 quanta/s. Thus a diffuse bipolar cell that collects from 10 cones (e.g., DB2 and DB3 in primate fovea; Calkins \& Sterling, 2007) receives about 7000 quanta/s. Eq. (3) indicates that each quantum carries only 0.002 bits. Thus, reversing direction, from the bridge entrance to the exit, the event rate declines, and bits per event rises (Fig. 2).

Notice that the ganglion cell, going from quanta to spikes, steps down the event rate by about 25 -fold. One advantage is that, compared to a spike, an excitatory quantum moves 1000-fold fewer cations and thus uses 1000-fold less ATP (Attwell \& Laughlin, 2001). Therefore, the ganglion cell's metabolic capacity remains in the plausible realm, and these considerations clarify that it is essential to step down the event rates before the spiking stage.

\section{Re-organizing information at the input to bipolar cells}

About 10 cones converge on a cone bipolar cell (Sterling, 2004). This figure varies by less than two-fold across the retina and across species. Apparently something about the fine correlations within natural images selects strongly for this degree of convergence. According to Eq. (1), this step potentially improves SNR at the bipolar cell by as much as $\sqrt{10}$. Thus a quite respectable quantity of contrast information is potentially available at the bipolar cell dendrites. But at this point, the information begins to be subdivided and redistributed unequally. That is, some bipolar cell types receive more information and others less.

One major step is to represent negative and positive contrasts separately. Transient dimming raises the cone's quantal rate, which depolarizes OFF bipolar cells; transient brightening lowers the cone's quantal rate, which depolarizes ON bipolar cells. Thus ON and OFF bipolar cells depolarize to opposite excursions of the cone's quantal rate. The mechanisms for opposite responses are well known: OFF dendrites express ionotropic glutamate receptors that open a cation channel; whereas ON dendrites express a metabotropic receptor that closes a cation channel (Sterling, 2004). Here is the advantage: if a single bipolar cell were employed to send the same total information as the two bipolar classes, the required modulation in quantal rate would more than double, due to the inherent inefficiency of higher rates (Eq. (3); von der Twer \& MacLeod, 2001).

Another advantage to separating the representation of positive and negative contrasts is that natural scenes contain more negative contrasts (Laughlin, 1981; Ratliff, Kao, Sterling \& Balasubramanian, unpublished; Richards, 1981). Thus an optimal representation should assign them more resources. Indeed, primate fovea expresses twice as many OFF diffuse bipolar cells as ON diffuse bipolar cells (Ahmad et al., 2003). Furthermore, an ON dendrite receives its cone quanta at the base of the invagination supplied by one ribbon (Vardi et al., 2000). But an OFF dendrite commonly occupies the space between two invaginations (Calkins \& Sterling, 2007; Haverkamp et al., 2000; Hopkins \& Boycott, 1995, 1997; Morigiwa \& Vardi, 1999). Such an OFF dendrite thus receives its cone quanta from two ribbons-sensing twice the quantal rate (DeVries et al., 2006; Klug, Sterling, \& Schein, unpublished). In short, there are more OFF bipolar cells than ON, and some OFF 
cells collect more information. This predicts that certain OFF diffuse bipolar cells should express more ribbon outputs than $\mathrm{ON}$ cells-but this point remains for future studies.

Another strategy to improve efficiency via lower quantal rates is to divide the temporal bandwidth. Both ON and OFF classes use this strategy. For example, in ground squirrel one high frequency type of OFF bipolar cell expresses AMPA receptors with large, fast currents that resensitize rapidly following a quantum; whereas two low frequency types express kainate receptors with smaller, slower currents that recover slowly and integrate over longer times (DeVries, 2000; DeVries et al., 2006). Similarly, in monkey fovea the AMPAexpressing type, DB3, locates many of its dendrites between ribbons where, as noted, they see quanta at higher rates than the kainate-expressing type, DB2. DB3 not only collects more quanta at the input, it also expresses more ribbons at its output (1.4-fold more than DB2) and is more numerous (1.5-fold) - so the DB3 array provides 2.1-fold more ribbon outputs (Calkins \& Sterling, 2007).

In summary, the bipolar arrays rigorously condense and rearrange their information so as to drastically reduce the mean rate of stochastic events. This is crucial because a ganglion cell cannot sustain spiking at the rates used by a cone. And even were this possible, it could not afford the metabolic cost.

Bipolar cells accomplish the critical condensation by sensing the input stream unequally. For example, we already calculated that under photopic conditions, a mammalian cone releases about 700 quanta/s. A monkey ON midget bipolar samples only 15 ribbons of a foveal cone's 20 ribbons (Calkins et al., 1996; Chun et al., 1996) and thus senses only about 500 quanta/s. But diffuse bipolar cells (DB2, DB3) sample on average 20 ribbons from each of 10 cones (Hopkins \& Boycott, 1995, 1997) and thus sense about 7000 quanta/s. One type of diffuse cell (DB3), half of whose dendrites sense quanta from 2 ribbons simultaneously (Calkins \& Sterling, 2007), will sense still more quanta, about 14000 quanta/s, nearly 30 -fold more than a midget cell.

Bipolar types that sense more quanta at the input (thus collecting more information) provide more ribbons at their outputs (Fig. 3). For example, the numbers of ribbon outputs from the midget bipolar cell, DB2, and DB3 are respectively 30, 48, and 69 (Calkins et al., 1994; Calkins \& Sterling, 2007; Klug et al., 2003). Now we address the final question: what is the maximum versus natural release rates from bipolar terminals onto ganglion cells?

\section{Maximum release rates from isolated bipolar terminals}

The "giant" goldfish bipolar terminal contains about 50 ribbons (Fig. 4). Each ribbon docks about 10 vesicles to the presynaptic active zone and tethers about 70 vesicles in higher rows, more distant from the plasma membrane (Sterling \& Matthews, 2005). The isolated terminal, when rapidly depolarized, fuses vesicles with two kinetically distinct pools: a small transient pool fuses about 500 vesicles in about one millisecond, and a larger sustained pool fuses about 2000 vesicles over several hundred milliseconds (Llobet et al., 2003; Zenisek et al., 2000, 2002). That pool, once depleted, requires seconds to refill. At the scale of a single ribbon, the transient pool releases at about 10 vesicles, which corresponds to the vesicles docked on the membrane. The sustained pool fuses about 40 vesicles, which corresponds to most of those tethered in

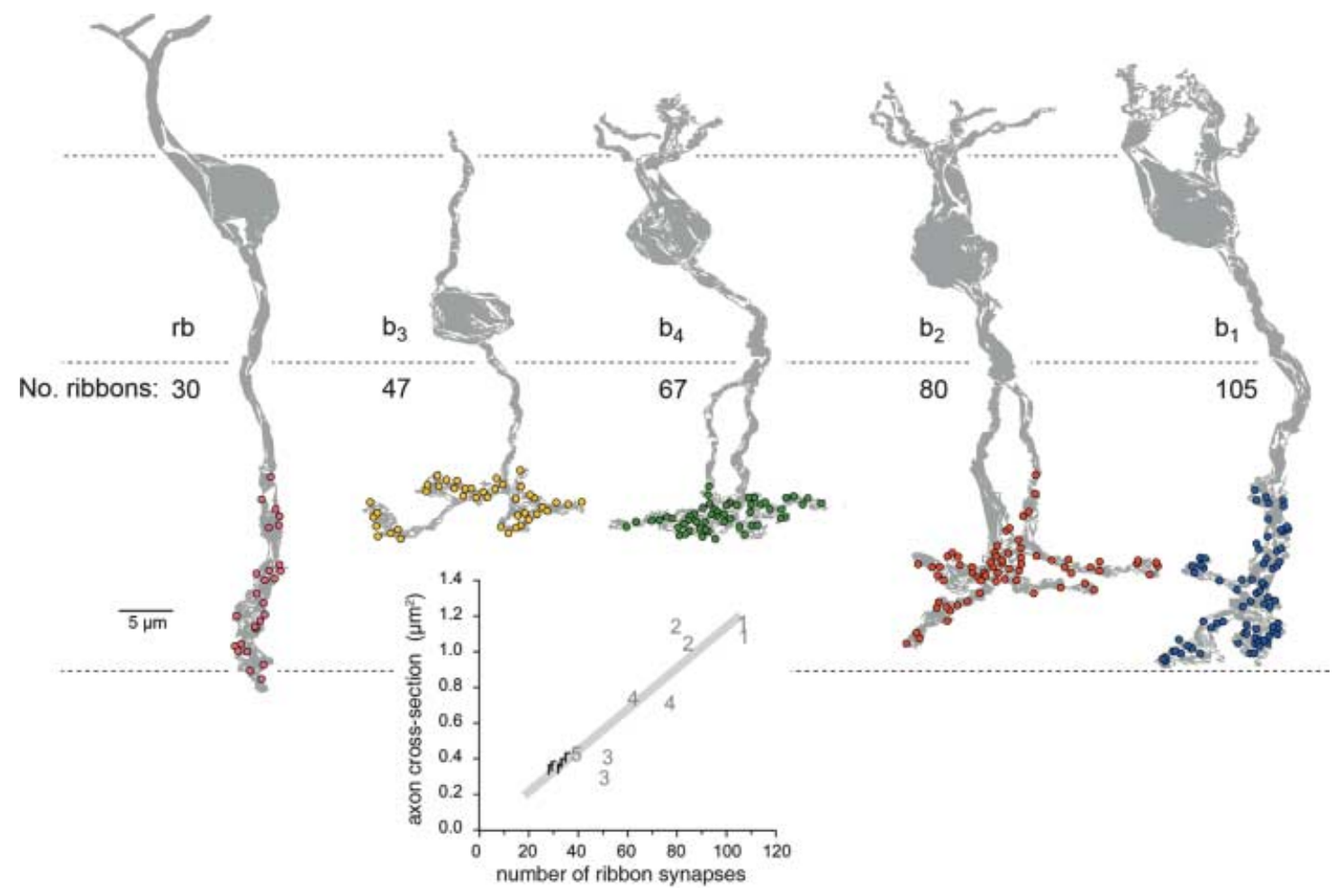

Fig. 3. Each type of ON bipolar cell expresses a different number of ribbon outputs (cat). The numbers correlate with temporal frequency, being lowest for the rod bipolar $(\mathrm{rb})$ and highest for $\mathrm{b}_{1}$, which is the transient cell that provides the main input to the brisk-transient ganglion cell. Axon cross-sectional area increases linearly with the number of ribbons. Data from Cohen and Sterling (1990). 

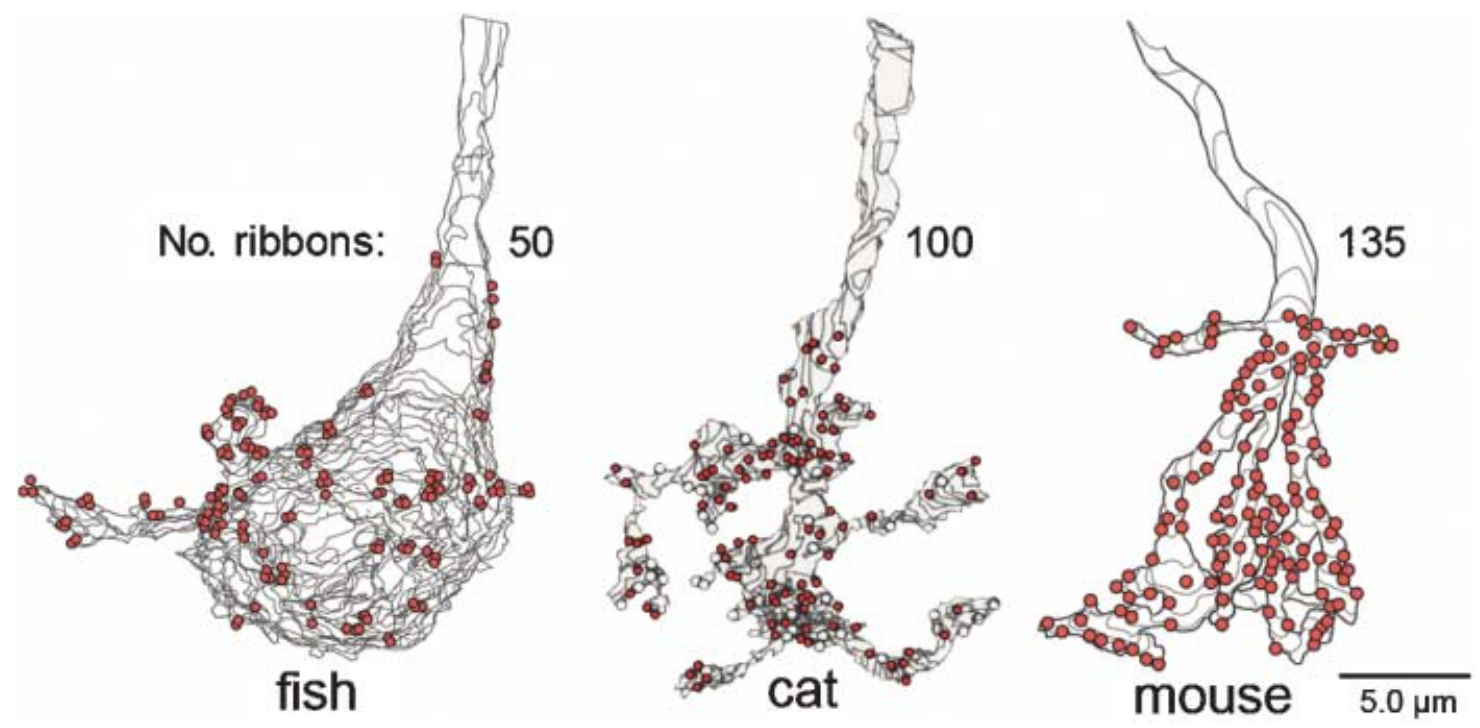

Fig. 4. Bipolar axon terminals exhibit similar scale across species. The goldfish cell is the "giant" ON bipolar (after von Gersdorff et al., 1996); the cat cell is the ON transient bipolar (after Cohen \& Sterling, 1990), and the mouse cell is an OFF bipolar, probably transient, (after Tsukamoto et al., 2001).

rows to the ribbon. Vesicles in the fish bipolar terminal diffuse rapidly to the ribbon (Holt et al., 2004), so as for the cone terminal, replenishing the ribbon is not a limiting step.

Mammalian bipolar terminals resemble fluted versions of the fish terminal, so their smaller cytoplasmic volumes contain fewer vesicles (Fig. 4). However, their ribbons are of similar size and number, and their transient and sustained pools are also similar: 7 and 35 quanta for rat (Singer \& Diamond, 2006); 40-60 vesicles for the combined pool in mouse (Zhou et al., 2006). Now we may ask how do these different kinetic pools compare to the release rates under natural conditions?

\section{Release rates from bipolar ribbons to light stimuli}

A brisk-transient ganglion cell in cat retina stimulated with a bright bar covering its receptive field center responds with a large transient depolarization caused by at most 10,000 quanta (Freed, 2000a). This cell type, at the retinal location where it was recorded, receives about 2000 ribbon synapses, mostly from a transient bipolar cell (Freed \& Sterling, 1988). Thus during the voltage transient, each ribbon releases about 5 quanta (Freed, $2000 a$ ). Consider that the ganglion cell transient response lasts about $100 \mathrm{~ms}$ - corresponding roughly to the combined kinetic pools (transient + sustained) of an isolated bipolar cell. Over that interval a drastic depolarization maximally releases about 50 vesicles from a ribbon, so we conclude that the releasable pool of a bipolar cell ribbon is about 10 -fold larger than its maximum response to a natural stimulus.

A brisk-sustained ganglion cell in cat retina stimulated by a bright bar responds with transient depolarization followed by a substantial sustained response $(4 \mathrm{mV})$ that can last for several seconds (Freed, 2000b). This response in a small central cell is caused by about 5000 quanta/s and in a larger peripheral cell, by about 45000 quanta/s. These rates divided by the respective numbers of ribbon contacts (260 and 2000); indicate that each ribbon can sustain about 20 quanta/s.
Although the critical experiments have not been done, we can roughly estimate release evoked by the lowest detectable contrast. A brisk-transient ganglion cell depolarizes detectably over several hundred milliseconds when the contrast of a center spot is only $1.5 \%$ (Dhingra \& Smith, 2004). Assuming that the threshold contrast causes an increment in release that just exceeds the noise, and given that release is Poisson (Freed, 2000a, 2005), then $1.5 \%$ of the number of quanta (n) equals $\sqrt{n}$. This requirement is satisfied when 4000 quanta increment to just above 4060. Because this ganglion cell receives approximately 5,000 ribbon synapses (Ying $\mathrm{Xu}$, unpublished), its membrane potential reaches response threshold when each ribbon releases less than 1 quantum-50-fold below the ribbon's capacity.

\section{Release rates from bipolar ribbons to naturalistic stimuli}

Release rates to naturalistic stimuli have been evaluated by measuring quantal rates received by a brisk-sustained ganglion cell evoked by photopic "white noise" (Fig. 5). This stimulus, presenting a rich variety of temporal frequencies, evokes spike patterns that resemble the responses to natural stimuli: spikes occur in bursts separated by silent intervals (Koch et al., 2006; Meister \& Berry, 1999). Each burst of spikes is triggered by a burst of quanta. The latter may contain up to 60 quanta, but the continuous rate (bursts + inter-burst periods) is about 100 quanta/s (Freed, 2005). Because the brisk-sustained cell receives about 3000-6000 ribbon contacts (guinea pig), each ribbon releases at most 0.02 quanta per burst and 0.03 quanta/s continuously. Thus, under quasi-natural conditions, the transient capacity of a ribbon, about 50 quanta per $100 \mathrm{~ms}$, exceeds the number in a burst by a factor of 2500. This safety factor does seem profligate, so what's going on?

First, considering ganglion cell design, it is desirable for the individual ribbon synapses to release relatively few quanta. This ensures that quanta will be locally rare and thus not saturate the driving force that generates the EPSC (Freed, 2000a). This is key 
A
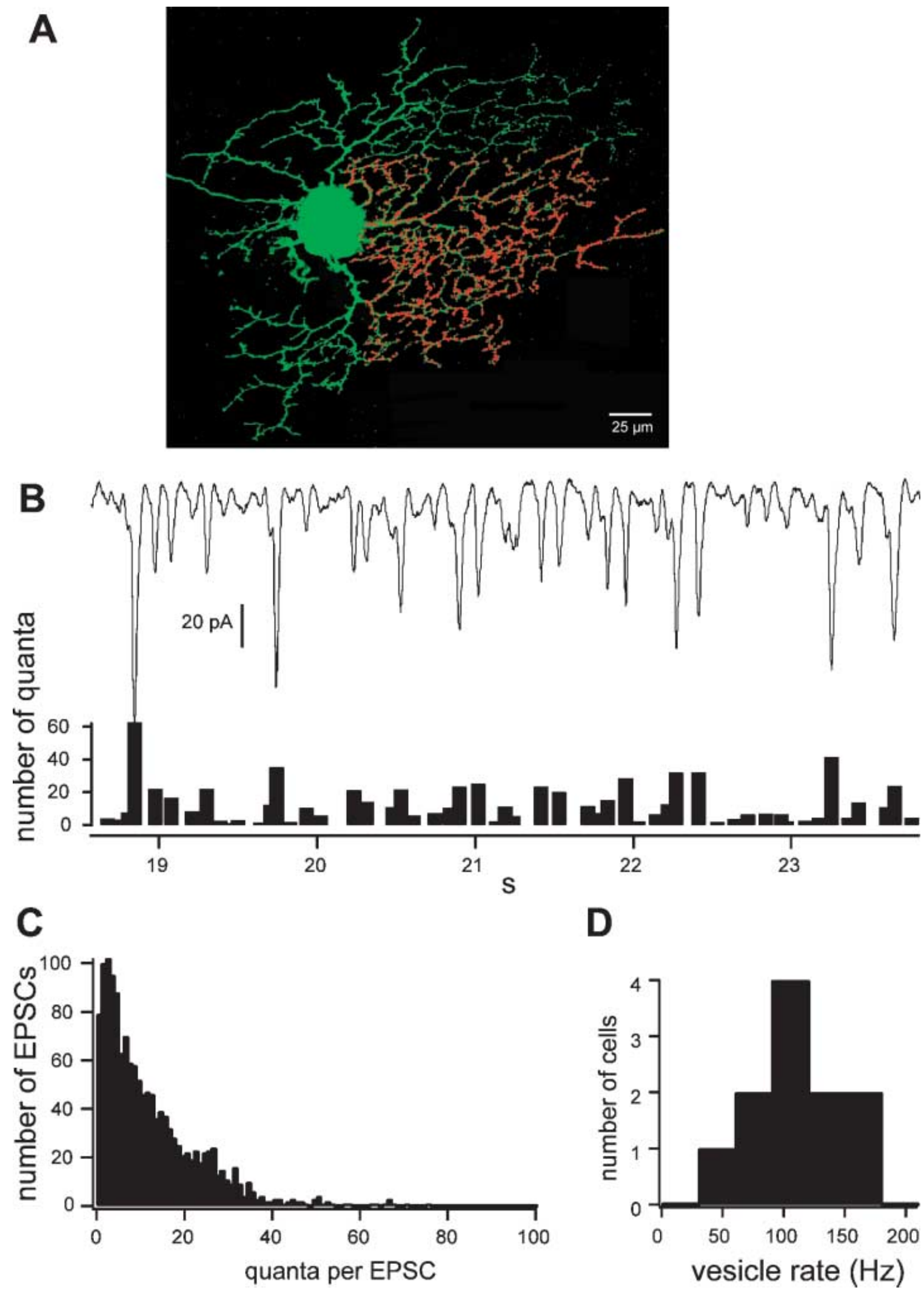

Fig. 5. (A) Brisk-sustained ganglion cell injected with Lucifer yellow (OFF cell from guinea pig). Ribbon synapses (red) were labeled with an antibody to kinesin, and their distribution was plotted for about one-third of the cell. Extrapolating, the whole cell would receive approximately 6000 synapses (Ying Xu, unpublished data). (B) Excitatory currents recorded under whole-cell voltage clamp from a brisk-sustained cell. Currents were evoked by white noise of photopic intensity and originated exclusively from bipolar cell synapses. EPSCs (downward deflections) comprise up to 60 quanta. (C) Distribution of quantal number in EPSCs from 11 brisk-sustained cells. (D) Distribution of average quantal rate from eight brisk-sustained cells. B-D are from Freed (2005). 
to maintaining linearity (Freed et al., 1992). On the other hand, the timing of both quanta and spikes to a repeated stimulus is quite precise: about 1-10 ms (Freed, 2005; Meister \& Berry, 1999). This, plus the fact that a burst of about 10 quanta is required to trigger a spike (Freed, 2005), raises the question: what mechanism allows 10 quanta to be released synchronously by 10 different ribbon synapses across the ganglion cell's dendritic field?

To achieve synchrony from a Poisson process requires high gain, and in biology high gain implies cooperativity (Koshland et al., 1982). Indeed, to release one vesicle at bipolar synapse requires four calcium ions (Heidelberger et al., 1994), and this in turn may require opening of several adjacent calcium channelsboth forms of cooperativity that would increase gain for large signals. By comparison, release at the cone synapse, which is continuous and greater by 2-3 orders of magnitude, shows virtually no cooperativity (Heidelberger et al., 2005). ${ }^{1}$

But high gain would tend to cause a high release rate. This implies that, following release of a single quantum, some mechanism(s) prevents further release. One mechanism is almost certainly feedback inhibition from an amacrine synapse (Lukasiewicz \& Shields, 1998) which can regulate quantal timing to within $10 \mathrm{~ms}$ (Freed et al., 2003). Also, a vesicle releases protons that inhibit subsequent release by blocking calcium channels, and this can regulate quantal timing to within 1-2 ms (Barnes et al., 1993; DeVries, 2000). Metabolic glutamate receptors on the bipolar terminal also mediate negative feedback, but they probably act too slowly to prevent release following one quantum (Awatramani \& Slaughter, 2001; Koulen et al., 1999).

\section{Conclusion}

The calculations presented here rest on various assumptions and approximations. Nevertheless, having undertaken them without prejudice, they support several key conclusions. First, across successive stages in this neural circuit, safety factors are on the order of 2-10. Thus, they resemble those in other tissues and systems. Their similarity across stages also accords with the principle of symmorphosis - that efficient design matches capacities across stages that are functionally coupled (Weibel, 2000). Second, we can now suggest why information traffic needs to be transformed from cone to ganglion cell (Fig. 2) - so that a spike train can transfer the essential information at lower event rates and thus save energy. Thus, we have clarified (hopefully) the actual purpose of this neural bridge.

\section{Acknowledgments}

Thanks to Ying $\mathrm{Xu}$ for contributing Figure 5A; also to David Calkins, Steven DeVries, Richard Kramer, Simon Laughlin, Gary Matthews, and Robert Smith for comments on the manuscript and to Sharron Fina for preparing it. Supported by EY00828, EY08124, and EY011105.

\section{References}

Ahmad, K.M., Klug, K., Herr, S., Sterling, P. \& Schein, S. (2003). Cell density ratios in a foveal patch in macaque retina. Visual Neuroscience 20, 189-209.

${ }^{1}$ The hair cell ribbon, where a single vesicle can evoke a spike, needs to release with extreme timing precision, and correspondingly shows extreme cooperativity requiring 5 calcium ions (Beutner et al., 2001).
Alexander, R.M. (1996). Optima for Animals. Princeton, NJ: Princeton University Press.

Attwell, D. \& Laughlin, S. (2001). An energy budget for signaling in the grey matter of the brain. Journal of Cerebral Blood Flow and Metabolism 21, 1133-1145.

Awatramani, G.B. \& Slaughter, M.M. (2001). Intensity-dependent, rapid activation of presynaptic metabotropic glutamate receptors at a central synapse. Journal of Neuroscience 21, 741-749.

Barnes, S., Merchant, V. \& Mahmud, F. (1993). Modulation of transmission gain by protons at the photoreceptor output synapse. Proceedings of the National Academy of Sciences USA 90, 10081-10085.

Beutner, D., Voets, T., Neher, E. \& Moser, T. (2001). Calcium dependence of exocytosis and endocytosis at the cochlear inner hair cell afferent synapse. Neuron 29, 681-690.

BURKHARDT, D.A. (2001). Light adaptation and contrast in the outer retina. Progress in Brain Research 131, 407-418.

Calkins, D.J., Schein, S., Tsukamoto, Y. \& Sterling, P. (1994). M and $\mathrm{L}$ cones in Macaque fovea connect to midget ganglion cells via different numbers of excitatory synapses. Nature 371, 70-72.

Calkins, D.J. \& Sterling, P. (2007). Microcircuitry for two types of achromatic ganglion cell in primate fovea. Journal of Neuroscience 27, 2646-2653.

Calkins, D.J., Tsukamoto, Y. \& Sterling, P. (1996). Foveal cones form basal as well as invaginating contacts with diffuse ON bipolar cells. Vision Research 36, 3373-3381.

Chrlovski, D.B., Schikorski, T. \& Stevens, C.F. (2002). Wiring optimization in cortical circuits. Neuron 34, 341-347.

Choi, S.-Y., Borghuis, B., Rea, R., Levitan, E.S., Sterling, P. \& Kramer, R.H. (2005). Encoding light intensity by the cone photoreceptor synapse. Neuron 48, 555-562.

Chun, M.-H., Grünert, U., Martin, P.R. \& Wässle, H. (1996). Thesynaptic complex of cones in the fovea and in the periphery of the macaque monkey retina. Vision Research 36, 3383-3395.

Cohen, E. \& Sterling, P. (1990). Demonstration of cell types among cone bipolar neurons of cat retina. Philosophical Transactions of the Royal Society of London B 330, 305-321.

de Ruyter van Steveninck, R. \& Laughlin, S.B. (1996). The rate of information transfer at graded-potential synapses. Nature 379, 642-645.

DeVries, S.H. (2000). Bipolar cells use kainate and AMPA receptors to filter visual information into separate channels. Neuron 28, 847-856.

DeVries, S.H., Li, W. \& SasziK, S. (2006). Parallel processing in two transmitter microenvironments at the cone photoreceptor synapse. Neuron 50, 735-748.

DeVries, S.H., QI, X., Smith, R.G., Makous, W. \& Sterling, P. (2002). Electrical coupling between mammalian cones. Current Biology 12, 1900-1907.

DhingRa, N.K. \& Smith, R.G. (2004). Spike generator limits efficiency of information transfer in a retinal ganglion cell. Journal of Neuroscience 24, 2914-2922.

Diamond, J. (1993). Evolutionary physiology. In: The logic of life. New York: Oxford University Press.

Esfahani, P., Schein, S., Klug, K., Tsukamoto, Y. \& Sterling, P. (1993). Characterization of L, M, and S cone pedicles in primate fovea. Society of Neuroscience 19, Abstract \#493.15.

Freed, M.A. (2000a). Rate of quantal excitation to a retinal ganglion cell evoked by sensory input. Journal of Neurophysiology 83, 2956-2966.

Freed, M.A. (2000b). Parallel cone bipolar pathways to ganglion cell use different rates and amplitudes of quantal excitation. Journal of Neuroscience 20, 3956-3963.

Freed, M.A. (2005). Quantal encoding of information in a retinal ganglion cell. Journal of Neurophysiology 94, 1048-1056.

Freed, M.A., Smith, R.G. \& Sterling, P. (1992). Computational model of the on-alpha ganglion cell receptive field based on bipolar circuitry. Proceedings of the National Academy of Sciences USA 89, 236-240.

Freed, M.A., Smith, R.G. \& Sterling, P. (2003). Timing of quantal release from the retinal bipolar terminal is regulated by a feedback circuit. Neuron 38, 89-101.

Freed, M.A. \& Sterling, P. (1988). The ON-alpha ganglion cell of the cat retina and its presynaptic cell types. Journal of Neuroscience $\mathbf{8}$, 2303-2320.

Haverkamp, S., Grünert, U. \& Wässle, H. (2000). The cone pedicle, a complex synapse in the retina. Neuron 27, 85-95.

Heidelberger, R., Heinemann, C., Neher, E. \& Matthews, G. (1994). Calcium dependence of the rate of exocytosis in a synaptic terminal. Nature 371, 513-515. 
Heidelberger, R., Thoreson, W.B. \& Witkovsky, P. (2005). Synaptic transmission at retinal ribbon synapses. Progress in Retinal and Eye Research 24, 682-720.

Holt, M., Cooke, A., Neef, A. \& Lagnado, L. (2004). High mobility of vesicles supports continuous exocytosis at a ribbon synapse. Current Biology 14, 173-183.

Hopkins, J.M. \& BoycotT, B. (1997). The cone synapses of cone bipolar cells of primate retina. Journal of Neurocytology 26, 313-325.

Hopkins, J.M. \& BoycotT, B.B. (1995). Synapses between cones and diffuse bipolar cells of a primate retina. Journal of Neurocytology $\mathbf{2 4}$, 680-694.

Klug, K., Herr, S., Ngo, I.-T., Sterling, P. \& Schein, S. (2003). Macaque retina contains an S-cone OFF midget pathway. Journal of Neuroscience 23, 9881-9887.

Koch, K., Mclean, J., Berry, M., Sterling, P., Balasubramanian, V. \& Freed, M.A. (2004). Efficiency of information transmission by retinal ganglion cells. Current Biology 14, 1523-1530.

Koch, K., Mclean, J., Segev, R., Freed, M.A., Berry, M.J.II., Balasubramanian, V. \& Sterling, P. (2006). How much the eye tells the brain. Current Biology 16, 1428-1434.

Koshland, D.E., JR., Goldbeter, A. \& Stock, J.B. (1982). Amplification and adaptation in regulatory and sensory systems. Science 217, 220-225.

Koulen, P., Kuhn, R., Wässle, H. \& Brandstätter, J.H. (1999). Modulation of the intracellular calcium concentration in photoreceptor terminals by a presynaptic metabotropic glutamate receptor. Proceedings of the National Academy of Sciences USA 96, 9909-9914.

Lagnado, L., Gomis, A. \& Job, C. (1996). Continuous vesicle cycling in the synaptic terminal of retinal bipolar cells. Neuron 17, 957-967.

LAUGHLIN, S. (1981). A simple coding procedure enhances a neuron's information capacity. Zeitschrift fur Naturforschung-Section CBiosciences 36, 910-912.

Laughlin, S.B. (1994). Matching coding, circuits, cells, and molecules to signals-general principles of retinal design in the fly's eye. Progress in Retinal and Eye Research 13, 165-196.

Laughlin, S.B. \& Hardie, R.C. (1978). Common strategies for light adaptation in the peripheral visual systems of fly and dragonfly. Journal of Comparative Physiology 128, 319-340.

Lennie, P. (2003). The cost of cortical computation. Current Biology 13, 493-497.

Li, W. \& DeVries, S.H. (2006). Bipolar cell pathways for color and luminance vision in a dichromatic mammalian retina. Nature Neuroscience 9, 669-675.

Llobet, A., Beaumont, V. \& Lagnado, L. (2003). Real-time measurement of exocytosis and endocytosis using interference of light. Neuron 40, 1075-1086.

Lukasiewicz, P. \& Shields, C.R. (1998). Different combinations of $\mathrm{GABA}_{\mathrm{A}}$ and $\mathrm{GABA}_{\mathrm{C}}$ receptors confer distinct temporal properties to retinal synaptic responses. Journal of Neurophysiology 79, 3157-3167.

Meister, M. \& Berry, M.J. (1999). The neural code of the retina. Neuron 22, 435-450.

Morigiwa, K. \& VARDI, N. (1999). Differential expression of ionotropic glutamate receptor subunits in the outer retina. Journal of Comparative Neurology 405, 173-184.

NELSON, R. (1977). Cat cones have rod input: a comparison of the response properties of cones and horizontal cell bodies in the retina of the cat. Journal of Comparative Neurology 172, 109-136.

Neves, G. \& Lagnado, L. (1999). The kinetics of exocytosis and endo- cytosis in the synaptic terminal of goldfish retinal bipolar cells. Journal of Physiology 515, 181-202.

Raviola, E. (1976). Intercellular junctions in the outer plexiform layer of the retina. Investigative Ophthalmology 15, 881-895.

Rea, R., Li, J., Dharia, A., Levitan, E.S., Sterling, P. \& Kramer, R.H. (2004). Streamlined synaptic vesicle cycle in cone photoreceptor terminals. Neuron 41, 755-766.

RICHARDS, W. (1981). A lightness scale from image intensity distributions. Applied Optics 21, 2569-2604.

Savage, G.L. \& Banks, M.S. (1992). Scotopic visual efficiency: Constraints by optics, receptor properties, and rod pooling. Vision Research 32, 645-656.

Singer, J.H. \& Diamond, J.S. (2006). Vesicle depletion and snaptic depression at a mammalian ribbon synapse. Journal of Neurophysiology $\mathbf{9 5}, 3191-3198$.

Smith, R.G. \& Sterling, P. (1990). Cone receptive field in cat retina computed from microcircuitry. Visual Neuroscience 5, 453-461.

Srinivasan, M.V., Laughlin, S.B. \& Dubs, A. (1982). Predictive coding: A fresh view of inhibition in the retina. Proceedings of the Royal Society (London) B 216, 427-459.

Sterling, P. (2004). How retinal circuits optimize the transfer of visual information. In The Visual Neurosciences. Cambridge, MA: MIT Press.

Sterling, P. \& Matthews, G. (2005). Structure and function of ribbon synapses. Trends in Neuroscience 28, 20-29.

Thoreson, W.B., Rabl, K., Townes-Anderson, E. \& Heidelberger, R. (2004). A highly Ca2+-sensitive pool of vesicles contributes to linearity at the rod photoreceptor ribbon synapse. Neuron 42, 595-605.

Tsukamoto, Y., Morigiwa, K., Ueda, M. \& Sterling, P. (2001). Microcircuits for night vision in mouse retina. Journal of Neuroscience 21, $8616-8623$.

Vardi, N., Duvoisin, R.M., Wu, G. \& Sterling, P. (2000). Localization of mGluR6 to dendrites of ON bipolar cells in primate retina. Journal of Comparative Neurology 423, 402-412.

VON DER Twer, T. \& MacLeod, D.I.A. (2001). Optimal nonlinear codes for the perception of natural colors. Network 12, 395-407.

von Gersdorff, H. \& Matthews, G. (1994). Dynamics of synaptic vesicle fusion and membrane retrieval in synaptic terminals. Nature 367, 735-739.

von Gersdorff, H., Sakaba, T., Berglund, K. \& Tachibana, M. (1998). Submillisecond kinetics of glutamate release from a sensory synapse. Neuron 21, 1177-1188.

von Gersdorff, H., Vardi, E., Matthews, G. \& Sterling, P. (1996). Evidence that vesicles on the synaptic ribbon of retinal bipolar neurons can be rapidly released. Neuron 16, 1221-1227.

WeIBEL, E.R. (2000). Symmorphosis. Cambridge, MA: Harvard University Press.

Zenisek, D., Steyer, J.A. \& Almers, W. (2000). Transport, capture and exocytosis of single synaptic vesicles at active zones. Nature $\mathbf{4 0 6}$, 849-854.

Zenisek, D., Steyer, J.A., Feldman, M.E. \& Almers, W. (2002). A membrane marker leaves syanptic vesicles in milliseconds after exocytosis in retinal bipolar cells. Neuron 35, 1085-1097.

Zhou, Z.Y., Wan, Q.F., ThaKur, P. \& Heidelberger, R. (2006). Capacitance measurements in the mouse rod bipolar cell identify a pool of releasable synaptic vesicles. Journal of Neurophysiology 96, 25392548 . 